\title{
Bacterial and Lyme Arthritis
}

\author{
John J. Ross, $M D^{*}$, and Linden T. Hu, MD
}

\author{
Address \\ *Division of Infectious Diseases, Caritas Saint Elizabeth's \\ Medical Center, 736 Cambridge Street, Boston, MA 02135, USA. \\ E-mail: jrossmd@cchcs.org
}

Current Infectious Disease Reports 2004, 6:380-387

Current Science Inc. ISSN I523-3847

Copyright (C) 2004 by Current Science Inc.

Septic arthritis is an infectious disease emergency, requiring aggressive joint drainage and prompt antibiotic therapy. Because age and joint damage are major risk factors, septic arthritis may become more common as the baby-boom generation reaches senescence. Bacteremic joint infections have a high mortality, and long-term disability from joint damage is common. Lyme arthritis has a negligible mortality and low morbidity but causes disproportionate popular anxiety. Most patients with Lyme arthritis respond well to oral antibiotic regimens and recover completely. A few patients develop antibiotic-resistant Lyme arthritis, which is probably autoimmune in nature and responds to immunosuppressive therapy. This paper reviews the presentation, diagnosis, and management of bacterial and Lyme arthritis. Septic arthritis caused by Streptococcus pneumoniae and group B streptococci may be increasing in prevalence. Prospective studies are required to determine whether there is any benefit of surgical or arthroscopic joint drainage in septic arthritis, compared with serial arthrocentesis, and to determine the role of corticosteroid therapy in septic arthritis in adults, if any.

\section{Introduction}

Septic arthritis is an infectious disease emergency, with significant morbidity and mortality. Because increasing age and pre-existing joint pathology are major risk factors, septic arthritis may become increasingly common as the baby-boom generation ages. In contrast, Lyme arthritis has a negligible mortality and low morbidity but has caused anxiety in the public mind (at times bordering on hysteria). This paper reviews the clinical, diagnostic, and therapeutic aspects of these two conditions.

\section{Pathogenesis}

Septic arthritis usually arises from hematogenous spread. Synovial joints lack basement membranes and are susceptible to bacteremic seeding [1]. Minute breaks in skin or mucous membranes may allow staphylococci and streptococci initial access to the bloodstream. Gramnegative septic arthritis generally arises from bacteremia from another infected site, such as the urinary tract. Occasionally, septic arthritis results from penetrating trauma, such as human or animal bites, stepping on nails, or errant injection drug use. Penetrating injury is the usual means of infection of the small joints of the hands and feet [2]. Rarely, therapeutic joint injection with corticosteroids may be complicated by septic arthritis.

Gram-positive organisms cause septic arthritis much more often than do gram-negative organisms. Although enteric gram-negative rods account for $43 \%$ of community-acquired bacteremias, they cause only $10 \%$ of septic arthritis $[3,4 \bullet]$. This may relate to the superior ability of gram-positive organisms to bind connective tissue and extracellular matrix proteins. Staphylococcus aureus, the most common cause of septic arthritis, produces several surface adhesins that bind extracellular matrix proteins, known as microbial surface components recognizing adhesive matrix molecules (MSCRAMMs). Staphylococcal strains defective in MSCRAMMs are less able to provoke septic arthritis in animal models [5].

Joint damage in septic arthritis results from bacterial invasion and the host response. Bacterial enzymes and toxins are directly injurious to cartilage. Neutrophils produce reactive oxygen species and lysosomal proteases. Cytokines promote activation of host matrix metalloproteinases, resulting in autodigestion of cartilage [ $6 \bullet$ ]. Tissue ischemia also plays a role. Cartilage is avascular and highly dependent on diffusion of oxygen and nutrients from the synovium. Joint pressure increases as purulent exudate accumulates within. Synovial blood flow is tamponaded, and cartilage anoxia results [7].

\section{Risk Factors}

The primary risk factor for septic arthritis is a pre-existing joint abnormality. Up to $47 \%$ of patients have an underlying joint disease [2]. These include rheumatoid arthritis, osteoarthritis, gout, pseudogout, recent trauma, prior joint surgery, and systemic lupus erythematosus. Of these, rheumatoid arthritis is the most common and is associated with worse outcomes.

Patients with rheumatoid arthritis are at high risk for septic arthritis from a combination of prior joint damage, immunosuppressive medications, and loss of skin integrity. Periarticular infection may result in sinus tracts, bursitis, and rupture of synovial cysts. Polyarticular disease 
is common, functional outcomes are worse, and mortality is high in rheumatoid arthritis patients with septic joints $[8,9]$. Diagnosis is often delayed because of the confusion of septic arthritis for a flare of rheumatoid arthritis. Septic arthritis may also be missed in patients with other chronic arthritic conditions such as gout [10], and a high index of suspicion for septic arthritis should be maintained in patients with other rheumatologic conditions.

Other conditions predisposing to septic arthritis can be categorized into those relating to a loss in skin integrity, such as psoriasis, eczema, skin ulcers, and injection drug abuse, and those that affect the ability to combat infection, such as diabetes mellitus, renal failure, cirrhosis, and immunosuppressive therapies. A recently described risk factor is anti-inflammatory therapy with tumor necrosis factor blockers, with poor outcomes in reported patients [11]. However, a significant proportion of patients with septic arthritis, up to $22 \%$ in some studies, has no medical risk factors and no underlying joint disease [12•].

\section{Clinical Syndromes and Organisms Clinical features}

The typical patient with septic arthritis presents after a few days of progressive symptoms with a warm, swollen, and exquisitely painful joint. However, the diagnosis of septic arthritis is often not obvious. High-grade fever is only present in $58 \%$ of patients [12•], although $90 \%$ have at least low-grade fever [13]. Serum leukocytosis is present in only $50 \%$ to $60 \%$ of patients $[12 \bullet, 13]$. Joint pain is blunted in the immunosuppressed, such as the rheumatoid arthritis patient on corticosteroids, resulting in delayed diagnosis and greater complications $[9,14]$.

Predictors of mortality in a multivariate analysis include age 65 years or more, confusion at presentation, and polyarticular disease. Predictors of joint damage include age 65 years or more, diabetes mellitus, and infection with $\beta$-hemolytic streptococci [12•].

\section{Joint distribution}

The knee is the principal target of bacterial septic arthritis. Forty-five percent of septic arthritis cases in adults involve the knee [2]. Presumably, this is a consequence of the imperfect human adaptation to bipedal locomotion. The enormous stresses affecting the knee particularly predispose it to injury [15]. Other large joints of the appendicular skeleton, including the hip (15\%), ankle (9\%), elbow (8\%), wrist $(6 \%)$, and shoulder $(5 \%)$, are commonly involved in adults [2]. Involvement of joints of the axial skeleton, such as the sacroiliac and sternoclavicular joints, is infrequent, except in intravenous drug users [16]. Polyarticular disease is seen in approximately $10 \%$ to $20 \%$ of cases; it is more common with gonococcal, pneumococcal, group B streptococcal, and gram-negative septic arthritis. Polyarticular septic arthritis is usually asymmetric and involves an average of four joints. At least one knee is involved in $72 \%$ of cases.
Major risk factors are steroid therapy, rheumatoid arthritis, lupus, and diabetes mellitus [17].

\section{Organisms}

Staphylococcus aureus is the most common bacterial cause of septic arthritis, accounting for $44 \%$ of cases (Table 1 ). Only $46 \%$ of cases have an underlying focus of staphylococcal infection, such as cellulitis [18]. In the remainder, septic arthritis presumably arises as a consequence of transient bacteremia from a skin or mucous membrane source. Outcomes are often poor. Mortality ranges from $7 \%$ to $18 \%$, and osteomyelitis or poor joint function occurs in up to $27 \%$ to $46 \%[12 \bullet, 13]$. The ability of $S$. aureus to induce a vigorous host immune response by superantigen production may contribute to mortality and arthritis severity [19].

Group B streptococci (Streptococcus agalactiae) have recently emerged as invasive pathogens in the elderly, especially those with diabetes, cirrhosis, and neurologic disease [20]. At one center, group B streptococci increased from $1 \%$ of septic arthritis cases in the 1980 s to $6 \%$ in the 1990s [21]. Group B streptococci were responsible for $10 \%$ of septic arthritis in another recent study. Bacteremia was seen in $66 \%$, polyarticular disease in $32 \%$, and mortality was $9 \%[22 \bullet]$. Functional outcomes are typically poor in septic arthritis caused by group B streptococci and other $\beta$-hemolytic streptococci [12•]. The sternoclavicular and sacroiliac joints seem to be involved with disproportionate frequency [22•], although this could be the result of reporting bias.

Streptococcus pneumoniae may also be increasing in importance as a cause of septic arthritis, accounting for up to $8 \%$ to $10 \%$ of cases in some recent studies $[23,24]$. As with septic arthritis caused by group B streptococci, pneumococcal septic arthritis is notable for a high frequency of bacteremia and polyarticular disease. Only $50 \%$ have an underlying focus of pneumococcal disease, such as pneumonia. Mortality in adults is high (19\%), although functional outcomes are good in $95 \%$ of survivors. Drug resistance may be an increasing problem [4•].

Neisseria gonorrhoeae, once the leading cause of septic arthritis in young adults in the United States, has dwindled in importance since the 1980s. Safer sex in the AIDS era resulted in a 64\% decrease in gonorrhea from 1985 to 1997 [25]. However, recent increases in high-risk sexual behavior in young homosexual men, and rising rates of fluoroquinolone-resistant gonorrhea, suggest that epidemic gonorrhea could return to the United States.

Gonococcal septic arthritis is a distinct clinical syndrome, with a good prognosis. Seventy-five percent of cases occur in women; menses and pregnancy increase the risk of disseminated gonococcal infection [26]. Seventytwo percent of cases are polyarticular [18]. Patients may experience a fleeting and migratory polyarthritis, or a more conventional septic arthritis picture of several hot, swollen, and exquisitely tender joints. Knee involvement is most 
Table I. Organisms isolated in 2407 cases of septic arthritis

\begin{tabular}{|lc|}
\hline Organism & Isolates, $\boldsymbol{n}$ (\%) \\
\hline Staphylococcus aureus & $1066(44.3)$ \\
Streptococcus pyogenes & $183(7.6)$ \\
Streptococcus pneumoniae & $156(6.5)$ \\
Haemophilus influenzae & $104(4.3)$ \\
Mycobacterium tuberculosis & $101(4.2)$ \\
Escherichia coli & $91(3.8)$ \\
Coagulase-negative staphylococci & $84(3.5)$ \\
Neisseria gonorrhoeae & $77(3.2)$ \\
Streptococcus agalactiae & $69(2.9)$ \\
Pseudomonas aeruginosa & $36(1.5)$ \\
Neisseria meningitidis & $28(1.2)$ \\
Salmonella species & $25(1)$ \\
Other gram-negative rods & $110(4.6)$ \\
Other $\beta$-hemolytic streptococci & $104(4.3)$ \\
Polymicrobial & $33(1.4)$ \\
Fungi & $4(0.2)$ \\
Miscellaneous & $136(5.7)$ \\
\hline
\end{tabular}

(Adapted from Ross et al. [4•].)

common. The characteristic hemorrhagic pustules of disseminated gonococcal infection are found in $42 \%$ of patients, and tenosynovitis is found in $21 \%$. Urinary signs or symptoms are present in only $32 \%$. Gonococci are recovered from joint fluid in less than $50 \%$ of cases. This may relate partly to the difficulty in recovering these fastidious organisms from cultures but may also indicate that some cases of gonococcal arthritis are immunemediated [26]. Complete recovery is the rule with appropriate therapy, and sequelae are rare.

Gram-negative rods cause approximately $10 \%$ of septic arthritis in adults. Two major groups are at risk: elderly patients with comorbid medical conditions, and young intravenous drug users $[27,28]$. Outcomes are typically better in the latter group. Older studies suggested that the mortality of gram-negative septic arthritis was $25 \%$, with poor functional outcomes in $79 \%$ of survivors [13]. More recent data suggest that outcome of gram-negative septic arthritis in older patients may be relatively good with prompt diagnosis and aggressive therapy, with mortality rates of only $5 \%$, and poor functional outcomes in $32 \%$. Perhaps surprisingly, an underlying source of gram-negative septic arthritis, such as a urinary tract infection, is only found in $50 \%$ of older patients [29]. Gram-negative bacilli have waned in importance as a cause of septic arthritis in injection drug users in recent years (see following text).

Tuberculous septic arthritis is uncommon in the United States. Patients complain of chronic, indolent joint pain, with an average duration of symptoms before diagnosis of 20 months. Sinus formation is seen in $25 \%$ to $50 \%$ of patients. Only $50 \%$ have systemic symptoms such as fever and sweats, and only $50 \%$ have findings on chest radiograph suggestive of tuberculosis. The most sensitive diagnostic test is biopsy and culture of synovium. The yield of synovial fluid acid-fast smears is low (20\%), although synovial fluid cultures are positive in $80 \%$. Large joint monoarthritis is typical, with approximately equal involvement of knee, hip, wrist, and ankle. Dactylitis, or digital involvement, is more common with mycobacterial than with bacterial arthritis [30].

Brucellar septic arthritis is rare in developed countries but common in countries where unpasteurized dairy products are consumed. In the United States, most cases are seen in immigrants from Latin America and the Middle East. Involvement of the sacroiliac joint is seen in up to $54 \%$ of patients, for unclear reasons [31].

\section{Special patient populations}

Intravenous drug users

Before 1983, Pseudomonas aeruginosa caused $64 \%$ of reported septic arthritis in intravenous drug users, with S. aureus responsible for only $10 \%$. After 1983, the roles of these pathogens were reversed: $P$. aeruginosa resulted in only $9 \%$ of septic arthritis in intravenous drug users, whereas S. aureus caused $71 \%$ [16]. This switch is explained by shifting patterns of intravenous drug use. In 1983, in response to an epidemic of pentazocine abuse, the manufacturer coformulated it with the narcotic antagonist naloxone. With oral administration, naloxone is rapidly inactivated, but when injected, it negates the intoxicating effect of pentazocine. Thereafter, heroin-supplanted pentazocine is the drug of choice among injection drug users [32].

Intravenous drug users often inject drugs with the nearest water supply, such as toilet water or puddles in an alleyway. In one study, $81 \%$ of injection drug users reported injecting in public toilets in the preceding 6 months, and $80 \%$ injected in the street [33]. Unlike pentazocine, which dissolves in water at room temperature, heroin must be heated to dissolve, typically by boiling in a spoon held over a flame. This process of "cooking" reduces contamination with environmental bacteria, such as Pseudomonas species. However, the risk of staphylococcal infection is equivalent with heroin and pentazocine abuse. "Cooking" does not decrease the risk of staphylococcal infection because the source of bacteria is likely a colonized skin site, rather than an environmental water source [34].

Septic arthritis in intravenous drug users often affects joints containing intra-articular cartilage, such as the pubic symphysis, and the sternoclavicular and sacroiliac joints. These joints are uncommonly infected in other patients with septic arthritis $[16,35,36]$. The progressive sclerosis and calcification with aging of these joints may decrease the risk of infection in the predominantly older population at highest risk for septic arthritis, compared with the younger patients who acquire septic arthritis from intravenous drug use. The sternoclavicular joint may account for up to $17 \%$ of septic arthritis in intravenous drug users. This joint is likely infected from 
phlebitis or valvulitis of the underlying subclavian vein, after injection of contaminated drugs into the upper extremity [36].

\section{Children}

In pediatric septic arthritis, knee involvement is not as preponderant as it is in adults. The knee and hip are infected in one third of cases each in children [37]. Staphylococci and streptococci are responsible for most cases. Haemophilus influenzae type b septic arthritis is now uncommon because of the protection provided by conjugate vaccines [38].

\section{Prosthetic joints}

The diagnosis and therapy of prosthetic joint infection have been reviewed in this journal recently by Zimmerli [39].

\section{Diagnosis}

Gram's stain and culture of synovial fluid should be routinely obtained in any case of undiagnosed arthritis. However, Gram's staining of synovial fluid is insensitive for the diagnosis of septic arthritis. Gram's stain is positive in $71 \%$ of gram-positive septic arthritis cases [13], $40 \%$ to $50 \%$ of cases of gram-negative septic arthritis [27-29], and less than $25 \%$ of cases of gonococcal septic arthritis [1].

It is recommended to treat patients empirically for septic arthritis when synovial fluid leukocyte counts exceed 100,000 cells $/ \mathrm{mm}^{3}$, although gout and pseudogout may also cause leukocyte counts of this magnitude [40]. Unfortunately, lower leukocyte counts do not exclude septic arthritis. As many as $50 \%$ of patients with septic arthritis have synovial fluid leukocyte counts less than 28,000 cells $/ \mathrm{mm}^{3}$ [41], and immunosuppressed patients may lack synovial leukocytosis altogether.

Synovial chemistry tests, such as glucose and protein, are not useful in the diagnosis of septic arthritis [42].

Blood cultures should be obtained in all patients with suspected septic arthritis. At least one third of patients with septic arthritis have associated bacteremia. In up to $14 \%$ of patients, a bacteriologic diagnosis is made only on the basis of blood cultures [18,43].

Serologic testing for Lyme disease should be obtained from patients with undiagnosed inflammatory arthritis in endemic areas, particularly if Gram's stain and culture of synovial fluid results are negative.

The diagnosis of gonococcal septic arthritis may be difficult. Less than $50 \%$ of synovial fluid culture results are positive, even when appropriately subcultured onto chocolate agar. The diagnosis is often made presumptively, on the basis of a clinical syndrome consistent with disseminated gonococcal infection and isolation of N. gonorrhoeae from cultures of the cervix, urethra, rectum, or oropharynx. Bacteremia is uncommon in disseminated gonococcal infection, despite the frequency of polyarticular involvement $[1,18,26]$.
The yield of cultures for fastidious organisms, such as nutritionally variant streptococci, may be improved by inoculation of synovial fluid into blood culture bottles [44].

\section{Therapy and Outcomes}

In general, because synovial fluid tests lack precision for septic arthritis, the clinical threshold for initiating antibiotics should be low. Empiric therapy should be directed toward likely organisms based on epidemiology and host factors. However, because septic arthritis is so rapidly destructive, broad-spectrum antibiotics are usually warranted until culture data are available. If Gram's stains of synovial fluid are negative, cefazolin is a reasonable initial choice for empiric coverage of suspected septic arthritis in patients at low risk for methicillin-resistant S. aureus (MRSA) and gonorrhea, providing staphylococcal, streptococcal and limited gram-negative coverage [45]. Patients at higher risk for gram-negative infection, such as the elderly and immunocompromised, could be treated with cefepime as a broad-spectrum single agent active against streptococci, methicillin-sensitive $S$. aureus, and gram-negative bacilli.

Intravenous drug users with septic arthritis may be covered empirically with vancomycin and cefepime, for MRSA and Pseudomonas species. Vancomycin is also reasonable empiric therapy for other patients with MRSA risk factors, such as hemodialysis, diabetes, recent nursing home admission, or recent or current hospitalization [46]. Certain areas of North America have experienced outbreaks of community-acquired MRSA infection, and it would be reasonable to initiate empiric therapy for MRSA in patients with septic arthritis in these regions [47]. There are favorable but very limited data supporting the use of linezolid as an alternative agent for MRSA septic arthritis [48].

Combination therapy with vancomycin and cefepime, or another agent with broad gram-negative activity, should be administered for critically ill patients with septic arthritis. Septic arthritis associated with human or animal bites should be treated with agents active against oral flora, such as ampicillin/sulbactam. Sexually active patients with clinical syndromes suggestive of disseminated gonococcal infection should receive ceftriaxone.

Once the causative organism has been identified, therapy should be tailored appropriately. Highly resistant gramnegative bacilli, such as Pseudomonas species, are best treated with the combination of a $\beta$-lactam drug with an aminoglycoside or a quinolone. Data on duration of therapy are scanty. In general, we would recommend courses of therapy for septic arthritis of at least 3 weeks, which may include a period of step-down oral therapy. Gonococcal septic arthritis can be treated with 2 weeks of therapy; patients treated with ceftriaxone should be monitored for the possible development of biliary sludging.

Septic arthritis is managed with prompt institution of appropriate antibiotics in combination with drainage 
of the joint by surgery, arthroscopy, or arthrocentesis. Joint drainage decompresses the joint, improves blood flow, and removes bacteria, toxins, and proteases. Arthrocentesis should be repeated daily until effusions resolve and culture results are negative. There is general agreement that surgical drainage is indicated for septic arthritis of the hip, failure to respond after 5 to 7 days of antibiotics and arthrocentesis, and soft-tissue extension of infection. The shoulder joint should be drained surgically or with radiologic guidance $[49,50]$. Retrospective data suggest that patients with rheumatoid arthritis have better functional outcomes with surgical management [8].

No good data show a superiority of surgical drainage over arthrocentesis. In fact, one meta-analysis, and a more recent retrospective study, demonstrated better functional outcomes with arthrocentesis compared with surgery, although mortality was higher in patients treated with arthrocentesis $[12 \bullet, 51]$. These differences are probably explained by patient selection. Patients in poor overall condition are unlikely to be surgical candidates, whereas otherwise stable patients with severe septic arthritis are more likely to undergo surgical drainage. Randomized, clinical trials of arthrocentesis compared with surgical or arthroscopic drainage are needed.

A recent study showed a significant benefit of dexamethasone therapy in preventing disability in children with septic arthritis, but no data exist to recommend its use in adults [52].

Aggressive rehabilitation is essential to prevent joint contractures and muscle atrophy. Patients should be mobilized with passive and active range-of-motion exercises as soon as pain allows. Although earlier reviews recommended joint immobilization in the acute phase of infection [1], in animal models of septic arthritis, more cartilage degeneration and adhesions were seen in immobilized animals, compared with animals treated with continuous passive motion devices [53].

\section{Lyme Arthritis \\ Pathogenesis}

Lyme arthritis differs significantly from bacterial septic arthritis in tempo and outcome of disease [54••]. After the bite of an infected deer tick, Borrelia burgdorferi causes an expanding rash, known as erythema migrans. Spirochetes enter the bloodstream as early as 3 days after the development of erythema migrans and may seed the heart, central nervous system, and joints. $B$. burgdorferi has a predilection for joint tissue, which may be mediated by specific binding to proteoglycans and glycosaminoglycans $[55,56]$.

Unlike bacteria such as $S$. aureus and streptococci, $B$. burgdorferi does not produce proteases that degrade cartilage and bone $[57,58]$. The presence of $B$. burgdorferi results in only mild to moderate inflammation in the joint. The result is a slowly progressing arthritis, more akin to rheumatoid arthritis than classic septic arthritis. Histologic examination of joint tissue in Lyme arthritis typically reveals synovitis without prominent cartilaginous and bony erosions [59]. Cartilage erosions develop only after months to years of disease and appear to be mediated by the host inflammatory response. Synovial fluid from patients with Lyme arthritis shows elevations in numerous inflammatory cytokines, such as tumor necrosis factor- $\alpha$ and interleukin-1, and proteases such as matrix metalloproteinases -1 and -3 , which are also implicated in the pathogenesis of rheumatoid arthritis [60].

The delayed development of cartilaginous and bony erosions allows for most patients who are treated for Lyme arthritis to recover fully-even if treatment is not initiated until late in the course of disease. A small subset of antibiotic-treated patients develops persistent joint inflammation, despite negative polymerase chain reaction (PCR) for B. burgdorferi from joint fluid. This antibioticresistant Lyme arthritis is probably autoimmune. Patients with HLA-DRB $1 * 0401$ and HLA-DRB $1 * 0101$ have a higher incidence of antibiotic-resistant Lyme arthritis [61]. Molecular mimicry of a $B$. burgdorferi protein, outer surface protein $A$, with human lymphocyte function antigen A-1, has been suggested as a possible mechanism [62].

\section{Risk factors}

Risk factors for Lyme disease include residence in an endemic area and exposure to Ixodes ticks. The North American strain of B. burgdorferi, B. burgdorferi sensu stricto, is more likely to cause arthritis than are the European strains, Borrelia garinii and Borrelia afzelii. Specific borrelial proteins may relate to differences in strain arthritogenicity [63]. No specific host factors have been identified that predict arthritis after infection with $B$. burgdorferi.

\section{Clinical features}

Although arthralgias are common in acute Lyme disease (1-4 weeks after infection), true arthritis develops only later in the course of disease (weeks to months after infection). Approximately 50\% of patients not receiving early antibiotic therapy will experience at least one episode of arthritis [64].

At the time of development of arthritis, the nonspecific symptoms related to the host inflammatory response (headache, fever, myalgias) have usually resolved, and the patient presents with isolated joint pain and swelling. Lyme arthritis is usually monoarticular or oligoarticular. The knee is by far the most commonly involved joint. Large knee effusions are common, although knee movement is not particularly painful, unlike most episodes of bacterial septic arthritis. Other large joints may be involved, including shoulder, ankle, elbow, wrist, and hip. B. burgdorferi may also infect joints rarely involved in bacterial septic arthritis, such as the temporomandibular, interphalangeal, metacarpophalangeal, and metatarsophalangeal joints [65].

The clinical course of untreated Lyme arthritis is typically intermittent, with one or more relapses. One 
potential cause of the relapsing and remitting course is surface antigenic shifts resulting in temporary escape from immune regulation and allowing spirochete proliferation and increased inflammation $[66,67]$.

Response to antibiotic therapy is excellent, with most patients having total resolution of arthritis within 1 to 2 months. Approximately $7 \%$ to $10 \%$ of patients will continue to have arthritis after antibiotic therapy [65]. Inflammation in antibiotic-resistant Lyme arthritis is typically constant, rather than intermittent. Additional antibiotics are usually ineffective, but patients do respond to immune-modulating agents. Even without immunomodulatory drugs, symptoms wane over time, and almost all patients have resolution of symptoms within 5 years.

\section{Diagnosis}

Lyme arthritis should be suspected in patients with oligoarticular arthritis in endemic areas. A detailed history of recent symptoms, travel, and lifestyle exposures should be obtained. The diagnosis of Lyme arthritis usually relies on serologic testing. The shortcomings of enzymelinked immunosorbent assay and Western blotting for Lyme disease have been extensively reviewed elsewhere and are beyond the scope of this review $[54 \bullet \bullet, 68]$. Newer serologic tests, such as the C- 6 antibody test, may have greater specificity without loss of sensitivity, although their utility has not been fully established.

Synovial fluid should be obtained from all patients with suspected Lyme arthritis. Synovial fluid leukocyte counts in Lyme arthritis usually range from 1000 to 50,000 cells $/ \mathrm{mm}^{3}$. However, leukocyte counts more than 100,000 cells $/ \mathrm{mm}^{3}$ do not exclude Lyme arthritis. In antibioticresistant Lyme arthritis, synovial leukocyte counts are generally lower, ranging from 500 to 10,000 cells $/ \mathrm{mm}^{3}$. PCR for detecting DNA of B. burgdorferi, although not yet approved for clinical use, is a useful confirmatory test

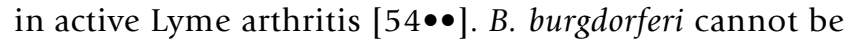
cultured from synovial fluid.

\section{Therapy}

Lyme arthritis can be successfully treated with oral or intravenous antibiotic regimens. In patients without evidence of neurologic disease, oral therapy is preferred because of lower cost and complication rates. Doxycycline $100 \mathrm{mg}$ twice daily is the agent of choice because of its excellent oral absorption, low cost, and anti-inflammatory activity as a matrix metalloproteinase inhibitor. Doxycycline is contraindicated in pregnant or breastfeeding women, and in young children, in whom it may cause tooth staining. Alternatives include amoxicillin, cefuroxime axetil, azithromycin, or clarithromycin; ceftriaxone is the intravenous drug of choice because of ease of administration. Patients with arthritis are generally treated for 4 weeks initially [69•].
A small proportion of patients do not respond to the initial course of antibiotics. Our approach is to perform repeat arthrocentesis, with synovial fluid PCR testing for B. burgdorferi DNA. Patients with a positive PCR test result should receive continued oral or intravenous antibiotics until symptoms resolve or PCR test results become negative. Patients with a negative PCR test result may also receive a single additional 4 weeks of antibiotic therapy. However, if symptoms persist in the face of repeatedly negative PCR test results, these patients should be treated for antibiotic-resistant Lyme arthritis.

Antibiotic-resistant Lyme arthritis responds well to steroids or methotrexate. Anticytokine therapy, such as tumor necrosis factor blocking agents, has been anecdotally reported to be effective in these patients and may eventually become the therapy of choice. Immunosuppressive medication is continued until patients have had complete suppression of arthritis for 3 months to 1 year, and then gradually tapered off. Synovectomy has a limited role for patients with severe arthritis unresponsive to all other measures.

\section{Conclusions}

Septic arthritis continues to be an important cause of morbidity and mortality in modern medicine. The epidemiology of septic arthritis has evolved in response to social factors (intravenous drug use and the aging of the population), medical practices that decrease host resistance (increased use of immunosuppressive agents), the emergence of new pathogens (B. burgdorferi), and regression of old ones (Mycobacterium tuberculosis and Brucella species). Outcomes are optimized by prompt therapy with appropriate antibiotics and joint drainage, followed by aggressive physical therapy and rehabilitation. Despite advances in our understanding of the pathogenesis of septic arthritis, outcomes have not improved much in the past several decades. In the future, cartilage regeneration and tissue engineering may assist in the recovery of joint function after septic arthritis.

\section{References and Recommended Reading}

Papers of particular interest, published recently, have been highlighted as:

- Of importance

-• Of major importance

1. Goldenberg DL, Reed JI: Bacterial arthritis. N Engl J Med 1985, 312:764-771.

2. Kaandorp CJ, Dinant HJ, van de Laar MA, et al.: Incidence and sources of native and prosthetic joint infection: a community based prospective survey. Ann Rheum Dis 1997, 56:470-475.

3. Haug JB, Harthug S, Kalager T, et al.: Bloodstream infections at a Norwegian university hospital, 1974-1979 and 1988-89: changing etiology, clinical features, and outcome. Clin Infect Dis 1994, 19:246-256. 
4. Ross JJ, Saltzman CL, Carling P, Shapiro DS: Pneumococcal septic arthritis: review of 190 cases. Clin Infect Dis 2003, 36:319-327.

This is a verbose but complete review. Pneumococcal septic arthritis is increasingly troublesome in regard to antibiotic resistance and may be becoming more prevalent.

5. Patti JM, Bremell T, Krajewska-Pietrasik D, et al.: The Staphylococcus aureus collagen adhesin is a virulence determinant in experimental septic arthritis. Infect Immun 1994, 62:152-161.

6. Shirtliff ME, Mader JT: Acute septic arthritis. Clin Microbiol Rev 2002, 15:527-544.

This is a good recent review of septic arthritis from a basic science and microbiology perspective.

7. Stevens CR, Williams RB, Farrell AJ, Blake DR: Hypoxia and inflammatory synovitis: observations and speculation. Ann Rheum Dis 1991, 50:124-132.

8. Gardner GC, Weisman MH: Pyarthrosis in patients with rheumatoid arthritis: a report of 13 cases and a review of the literature from the past 40 years. Am J Med 1990, 88:503-511.

9. Blackburn WD, Dunn TL, Alarcón GS: Infection versus disease activity in rheumatoid arthritis: eight years' experience. South Med J 1986, 79:1238-1241.

10. Ilahi OA, Swarna U, Hamill RJ, et al.: Concomitant crystal and septic arthritis. Orthopedics 1996, 19:613-617.

11. Baghai M, Osmon DR, Wolk DM, et al.: Fatal sepsis in a patient with rheumatoid arthritis treated with etanercept. Mayo Clin Proc 2001, 76:653-656.

12. Weston VC, Jones AC, Bradbury N, et al.: Clinical features and outcome of septic arthritis in a single UK Health District 1982-1991. Ann Rheum Dis 1999, 58:214-219.

This is one of the largest recent studies of septic arthritis cases. Mortality and morbidity were high, despite appropriate therapy in most patients.

13. Goldenberg DL, Cohen AS: Acute infectious arthritis. Am J Med 1976, 60:369-377.

14. Edwards SA, Cranfield T, Clarke HJ: Atypical presentation of septic arthritis in the immunosuppressed patient. Orthopedics 2002, 25:1089-1090.

15. Jones A, Doherty M: ABC of rheumatology: osteoarthritis. BMJ 1995, 310:457-460.

16. Brancós MA, Peris P, Miró JM, et al.: Septic arthritis in heroin addicts. Semin Arthritis Rheum 1991, 21:81-87.

17. Dubost JJ, Fis I, Denis P, et al.: Polyarticular septic arthritis. Medicine (Baltimore) 1993, 72:296-310.

18. Sharp JT, Lidsky MD, Duffy J, Duncan MW: Infectious arthritis. Arch Intern Med 1979, 139:1125-1130.

19. Bremell T, Tarkowski A: Preferential induction of septic arthritis and mortality by superantigen-producing staphylococci. Infect Immun 1995, 63:4185-4187.

20. Farley MM: Group B streptococcal disease in nonpregnant adults. Clin Infect Dis 2001, 33:556-561.

21. Dubost JJ, Saubrier M, De Champs C, et al.: No changes in the distribution of organisms responsible for septic arthritis over a 20 year period. Ann Rheum Dis 2002, 61:267-269.

22. Nolla JM, Gómez-Vaquero C, Corbella X, et al.: Group B Streptococcus (Streptococcus agalactiae) pyogenic arthritis in nonpregnant adults. Medicine (Baltimore) 2003, 82:119-127.

This paper reviews the clinical features of septic arthritis caused by the group B Streptococcus, an emerging pathogen in the elderly and immunocompromised.

23. Ryan MJ, Kavanagh R, Wall PG, Hazleman BL: Bacterial joint infections for England and Wales: analysis of bacterial isolates over a four year period. Br J Rheumatol 1997, 36:370-373.

24. Ispahani P, Weston VC, Turner DPJ, Donald FE: Septic arthritis due to Streptococcus pneumoniae in Nottingham, United Kingdom, 1985-1998. Clin Infect Dis 1999, 29:1450-1454.

25. Centers for Disease Control and Prevention: GonorrheaUnited States, 1998. MMWR Morb Mortal Wkly Rep 2000, 49:538-542.
26. O'Brien JP, Goldenberg DL, Rice PA: Disseminated gonococcal infection: a prospective analysis of 49 patients and a review of pathophysiology and immune mechanisms. Medicine (Baltimore) 1983, 62:395-406.

27. Goldenberg DL, Brandt KD, Cathcart ES, Cohen AS: Acute arthritis due to Gram-negative bacilli: a clinical characterization. Medicine (Baltimore) 1974, 53:197-208.

28. Bayer AS, Chow AW, Louie J, et al.: Gram-negative bacillary septic arthritis: clinical, radiographic, therapeutic, and prognostic features. Semin Arthritis Rheum 1977, 7:123-132.

29. Newman ED, Davis DE, Harrington TM: Septic arthritis due to Gram negative bacilli: older patients with good outcome. J Rheumatol 1988, 15:659-662.

30. Lee SH, Abramson SB: Infections of the musculoskeletal system by M. tuberculosis. In Tuberculosis. Edited by Rom WN, Garay SM. Boston: Little, Brown and Company; 1996:635-644

31. Gotuzzo E, Alarcon GS, Bocanegra TS, et al.: Articular involvement in human brucellosis: a retrospective analysis of 304 cases. Semin Arthritis Rheum 1982, 12:245-255.

32. Baum C, Hsu JP, Nelson RC: The impact of the addition of naloxone on the use and abuse of pentazocine. Public Health Rep 1987, 102:426-429.

33. Darke S, Kaye S, Ross J: Geographic injecting locations among injecting drug users in Sydney, Australia. Addiction 2001, 96:241-246.

34. Tuazon CU, Sheagren JN: Increased rate of carriage of Staphylococcus aureus among narcotic addicts. J Infect Dis 1974, 129:725-727.

35. Ross JJ, Hu LT: Septic arthritis of the pubic symphysis: review of 100 cases. Medicine (Baltimore) 2003, 82:340-345.

36. Ross JJ, Shamsuddin H: Sternoclavicular septic arthritis: review of 180 cases. Medicine (Baltimore) 2004, 83:139-148.

37. Krogstad P, Smith AL: Osteomyelitis and septic arthritis. In Textbook of Pediatric Infectious Disease, edn 4. Edited by Feigin RD, Cherry JD. Philadelphia: WB Saunders; 1998:683-704.

38. Luhmann JD, Luhmann SJ: Etiology of septic arthritis in children: an update for the 1990s. Pediatr Emerg Care 1999, 15:40-42.

39. Zimmerli W: Prosthetic joint infection: diagnosis and treatment. Curr Infect Dis Rep 2000, 2:377-379.

40. Fye KH: Arthrocentesis, synovial fluid analysis, and synovial biopsy. In Primer on the Rheumatic Diseases, edn 12. Edited by Klippel JH. Atlanta: Arthritis Foundation; 2001:138-144.

41. McCutchan HJ, Fisher RC: Synovial leukocytosis in infectious arthritis. Clin Orthop Rel Res 1990, 257:226-230.

42. Shmerling RH, Delbanco TL, Tosteson AN, Trentham DE: Synovial fluid tests. What should be ordered? JAMA 1990, 264:1009-1014

43. Cooper C, Cawley MI: Bacterial arthritis in an English health district: a 10 year review. Ann Rheum Dis 1986, 45:458-463.

44. Hepburn MJ, Fraser SL, Rennie TA, et al.: Septic arthritis caused by Granulicatella adiacens: diagnosis by inoculation of synovial fluid into blood culture bottles. Rheumatol Int 2003, 23:255-257

45. Fass RJ: Treatment of osteomyelitis and septic arthritis with cefazolin. Antimicrob Agents Chemother 1978, 13:405-411.

46. Salgado CD, Farr BM, Calfee DP: Community-acquired methicillin-resistant Staphylococcus aureus: a meta-analysis of prevalence and risk factors. Clin Infect Dis 2003, 36:131-139.

47. Naimi TS, LeDell KH, Como-Sabetti K, et al: Comparison of community-acquired and health-care associated methicillinresistant Staphylococcus aureus infection. JAMA 2003, 290:2976-2984.

48. Howden BP, Ward PB, Charles PG, et al.: Treatment outcomes for serious infections caused by methicillin-resistant Staphylococcus aureus with reduced vancomycin susceptibility. Clin Infect Dis 2004, 38:521-528.

49. Pioro MH, Mandell BF: Septic arthritis. Rheum Dis Clin North Am 1997, 23:239-258. 
50. Smith JW, Piercy EA: Infectious arthritis. Clin Infect Dis 1995, 20:225-231.

51. Broy SB, Schmid FR: A comparison of medical drainage (needle aspiration) and surgical drainage (arthrotomy or arthroscopy) in the initial treatment of infected joints. Clin Rheum Dis 1986, 12:501-522.

52. Odio CM, Ramirez T, Arias G, et al.: Double blind, randomized, placebo-controlled study of dexamethasone therapy for hematogenous septic arthritis in children. Pediatr Infect Dis $J$ 2003, 22:883-889.

53. Salter RB: The biologic concept of continuous passive motion of synovial joints: the first 18 years of research and its clinical application. Clin Orthop 1989, 242:12-25.

$54 . \bullet$ Steere AC: Lyme disease. N Engl J Med 2001, 345:115-125.

This overview is authoritative and comprehensive.

55. Leong JM, Robbins D, Rosenfeld L, et al.: Structural requirements for glycosaminoglycan recognition by the Lyme disease spirochete, Borrelia burgdorferi. Infect Immun 1998, 66:6045-6048.

56. Leong JM, Wang H, Magoun L, et al.: Different classes of proteoglycans contribute to the attachment of Borrelia burgdorferi to cultured endothelial and brain cells. Infect Immun 1998, 66:994-999.

57. Klempner MS, Noring R, Epstein MP, et al.: Binding of human plasminogen and urokinase-type plasminogen activator to the Lyme disease spirochete, Borrelia burgdorferi. J Infect Dis 1995, 171:1258-1265.

58. Coleman JL, Sellati TJ, Testa JE, et al.: Borrelia burgdorferi binds plasminogen, resulting in enhanced penetration of endothelial monolayers. Infect Immun 1995, 63:2478-2484.

59. Steere AC, Duray PH, Butcher EC: Spirochetal antigens and lymphoid cell surface markers in Lyme synovitis: comparison with rheumatoid synovium and tonsillar lymphoid tissue. Arthritis Rheum 1988, 31:487-495.
60. Lin B, Kidder JM, Noring R, et al.: Differences in synovial fluid levels of matrix metalloproteinases suggest separate mechanisms of pathogenesis in Lyme arthritis before and after antibiotic treatment. J Infect Dis 2001, 184:174-180.

61. Kalish RA, Leong JM, Steere AC: Association of treatmentresistant chronic Lyme arthritis with HLA-DR4 and antibody reactivity to OspA and OspB of Borrelia burgdorferi. Infect Immun 1993, 61:2774-2779.

62. Gross DM, Forsthuber T, Tary-Lehmann M, et al.: Identification of LFA- 1 as a candidate autoantigen in treatment-resistant Lyme arthritis. Science 1998, 281:703-706.

63. Wang G, Ojaimi C, Wu H, et al.: Disease severity in a murine model of lyme borreliosis is associated with the genotype of the infecting Borrelia burgdorferi sensu stricto strain. J Infect Dis 2002, 186:782-791.

64. Steere AC, Craft JE, Hutchinson GJ, et al.: The early clinical manifestations of Lyme disease. Ann Intern Med 1983, 99:76-82.

65. Steere AC, Schoen RT, Taylor E: The clinical evolution of Lyme arthritis. Ann Intern Med 1987, 107:725-731.

66. Zhang JR, Norris SJ: Genetic variation of the Borrelia burgdorferi gene vlsE involves cassette-specific, segmental gene conversion. Infect Immun 1998, 66:3698-3704.

67. Zhang JR, Hardham JM, Barbour AG, Norris SJ: Antigenic variation in Lyme disease borreliae by promiscuous recombination of VMP-like sequence cassettes. Cell 1997, 89:275-285.

68. Hu LT, Klempner MS: Update on the prevention, diagnosis, and treatment of Lyme disease. Adv Intern Med 2001, $46: 247-275$

69. Wormser GP, Nadelman RB, Dattwyler RJ, et al.: Practice guidelines for the treatment of Lyme disease. The Infectious Diseases Society of America. Clin Infect Dis 2000, 31:S1-S14. This set of treatment guidelines is based on a thorough review of the relevant literature. 